\title{
The Inheritance of Penicillin Titre in Crosses between Lines of Aspergillus nidulans Selected for Increased Productivity
}

\author{
By M. J. MERRICK* \\ Department of Genetics, University of Birmingham, B15 $2 T T$
}

(Received 2 June 1975)

SUMMAR Y

\begin{abstract}
Selection for increased penicillin production among the progeny of pairwise crosses between wild-type isolates of Aspergillus nidulans resulted in the production of high-titre strains. Three crosses were made between strains with increased titre derived from independent selection lines. Significant genetic variation was found among the progeny of each cross, indicating that different genes for increased titre had been selected in each line. Gene action was additive in each cross. Renewed selection from the progeny of one 'between-line' cross resulted in further increases in titre, over three successive generations.
\end{abstract}

\section{INTRODUCTION}

In the preceding paper (Merrick, I975) it was shown that selection for increased titre was effective in each of four independent selection lines in Aspergillus nidulans. After three, four or five generations of selection, titre had been increased by between 60 and $160 \%$ in each line and the available genetic variability considerably reduced.

The present paper describes investigations of the genetic architecture of these different selection lines. Two questions in particular were considered: whether the increases in titre obtained in the different lines were due to the selection of the same or different genes, and whether the gene action in selected strains remained predominantly additive, as was the case with unselected wild-type isolates (Merrick \& Caten, 1975a).

\section{METHODS}

General. Media, and methods of culture, genetic analysis and titre assessment were all as described by Merrick \& Caten (I975a).

Penicillin fermentations and assays. These were essentially as described by Merrick \& Caten (1975a). In assaying progeny from crosses between selection lines the number of replicate flasks per progeny strain was increased to three, and six flasks were used for each of the parental and control strains. This increased level of replication gives greater precision, and is particularly important when examining the data for evidence of epistasis (for a detailed discussion see Merrick \& Caten, I975b). A sample of 44 progeny strains was always used. For comparisons between generations all parental and progeny means were corrected against the control strains as described by Merrick (1975).

Selection procedure. During the first cycle of selection it proved impossible to cross the two selected progeny from the third generation in line 3 and this line had to be terminated (Merrick, 1975). The three remaining selection lines were each continued for four or five

\footnotetext{
* Present address: John Innes Institute, Colney Lane, Norwich NR4 7 UH .
} 
Table I. Analyses of variance for penicillin titre among progeny from crosses between high-titre selection lines in A. nidulans

\begin{tabular}{|c|c|c|c|c|c|c|c|c|c|}
\hline \multirow[b]{3}{*}{ Cross } & \multirow[b]{3}{*}{ Selected lines } & & & \multicolumn{6}{|c|}{ Item } \\
\hline & & \multicolumn{2}{|c|}{ Parental strains } & Betwe & progeny & \multicolumn{2}{|c|}{$\begin{array}{l}\text { Between assay } \\
\text { plates }\end{array}$} & \multicolumn{2}{|c|}{ Plates $x$ progeny } \\
\hline & & $P_{1} \times$ & $\mathbf{P}_{2}$ & D.F. & M.S. & D.F. & M.S. & D.F. & M.S. \\
\hline M50 & Line $2 \times$ line 4 & $\operatorname{Mi} 5.22 \times$ & MI $7 \cdot 26$ & 42 & $52 \cdot 46^{*}$ & 2 & 35.29 & 86 & 15.69 \\
\hline м60 & Line $1 \times$ line 2 & MI $4.36 / \mathrm{I} \times$ & MI9.36 & 43 & $62 \cdot 58^{*}$ & 2 & $38 \cdot 64$ & 82 & 26.51 \\
\hline M70 & Line $\mathrm{I} \times$ line 4 & MI $4.43 \times$ & $M 2 I \cdot 5 / 1$ & 43 & $75 \cdot 29^{*}$ & 2 & 14.67 & 86 & $25 \cdot 77$ \\
\hline
\end{tabular}

generations and crosses were then made between the lines in all possible combinations (Table I). In two of these crosses (M60 and M70) a white spore-colour mutation was used instead of the yellow markers used previously. This change was made because yellow mutations had been found to have a depressing pleiotropic effect on titre (Merrick \& Caten, I975a; Merrick, 1975). A high-titre green-spored strain was chosen from the fourth generation of line I (cross MI4) and the fifth generation of line 4 (cross M2I), and a white sporecolour mutation was induced in each strain using ultraviolet light. The two resulting whitespored strains MI4.36/I and M2I.5/I were then crossed to green-spored strains from the fifth generation of line 2 and the fourth generation of line I respectively. For the remaining cross (M50) a green-spored strain from the fifth generation of line 2 was crossed with a yellow-spored strain from the fifth generation of line 4 .

To examine whether further increases in titre could be obtained by combining favourable alleles from different selected lines, a second cycle of selection was initiated. The highesttitre green-spored and highest-titre yellow-spored progeny were selected from cross M5O and were crossed. The selection procedure was then identical to that of Merrick \& Caten

$975 a$ ) except that in the second generation of renewed selection the marker used for detection of hybrid perithecia was changed from a yellow to a white spore-colour mutation for the reasons discussed above. For this purpose the two highest-titre green-spored strains were selected from the first generation and a white mutant was induced in one of them. The white-spored and green-spored strains were then used as parents for the second generation. The second cycle of selection (line $2 \times 4$ ) was continued for two generations.

Contemporaneous comparison of selected strains. Throughout the selection programme strains selected as parents of the succeeding generation were stored on silica gel at $4{ }^{\circ} \mathrm{C}$. It was therefore possible to compare the titres of all these strains in a single experiment at the end of the programme. Four replicate flasks were used for each strain and the two control strains were also included. The mean parental titres for each generation, calculated from this experiment, were then compared with the respective progeny mean titres obtained in previous experiments. This comparison is based on the fact that the gene action has been shown to be predominantly additive for this character throughout the selection programme (Merrick \& Caten, I975a; Merrick, 1975). 


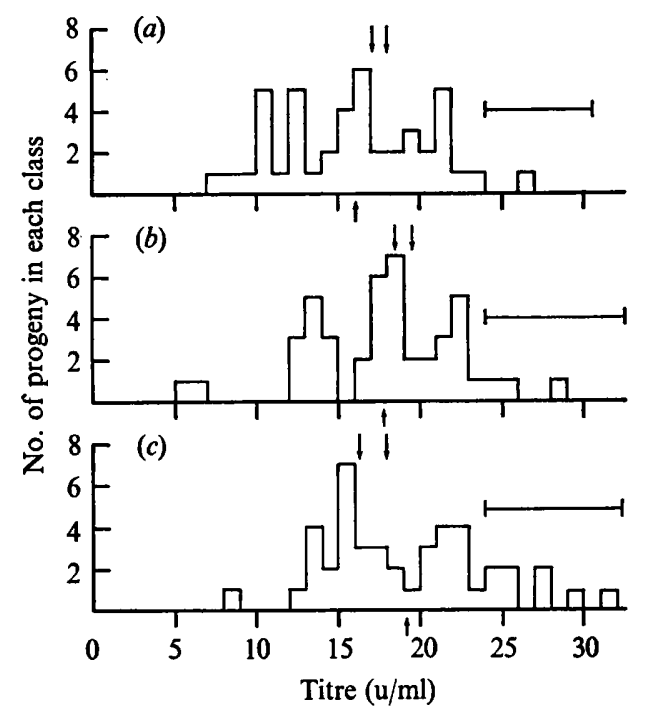

Fig. I. Distribution of progeny titres in three crosses between strains of $A$. nidulans selected for increased penicillin production. (a) $\mathrm{M} 50,(b) \mathrm{M} 60,(c) \mathrm{M} 70 . \mapsto$, Least significant difference $5 \%$; $\downarrow$. parental mean titres; $\uparrow$, progeny mean titre.

\section{RESULTS}

\section{Crosses between selected lines}

The methods used for analysis of these crosses were as described by Merrick \& Caten (I975a). The progeny titres were subjected to an analysis of variance and the following statistics were calculated: progeny mean titre; parental mean titre; ${\sigma_{E}}^{2}$, a measure of the environmental variation in the fermentation and the assay; $\sigma_{G}{ }^{2}$, a measure of the variation due to segregation of natural allelic differences affecting penicillin titre; $\sigma_{C}{ }^{2}$, a measure of variation associated with the spore-colour markers; $h^{2}$, the heritability of penicillin titre in each cross. The progeny and parental mean titres were compared to estimate $[i]$, the contribution of non-additive gene effects to the mean phenotype, in each cross.

The analyses of variance (Table I) indicated that there were significant differences between the high-titre strains from all three lines. Significant genetic variation was found among the progeny in each cross (Fig. I) and the estimates of ${\sigma_{\theta}}^{2}$ (Table 2) were as large or larger than those obtained in crosses between wild-type isolates (Merrick \& Caten, 1975a). In cross $\mathrm{M} 50$, where the genetic marker was a yellow spore-colour mutation, the mean titre of yellow-spored progeny was significantly lower than that of green-spored progeny, but in crosses $\mathrm{M} 60$ and $\mathrm{M70}$, where a white spore-colour marker was used, there was no significant marker effect and the estimate of ${\sigma_{C}}^{2}$ was zero (Table 2). In each cross the parental mean was not significantly different from the progeny mean (Fig. I) and hence the estimates of [i] (Table 2) were not significantly different from zero, indicating that the gene action was predominantly additive in each case.

\section{Renewed selection for increased titre}

The progeny mean titres in each generation were corrected against the control strain titres as described by Merrick (1975) to allow for variation in titre caused by uncontrollable environmental variation between experiments. The response in the second cycle was similar 
Table 2. Summary of data for inheritance of penicillin titre in three crosses between high-titre selection lines of $A$. nidulans

\begin{tabular}{|c|c|c|c|c|c|c|c|c|}
\hline \multirow[b]{2}{*}{ Cross } & \multicolumn{2}{|c|}{$\begin{array}{l}\text { Parental mean } \\
\text { titre }(\mathrm{u} / \mathrm{ml})\end{array}$} & \multirow{2}{*}{$\begin{array}{c}\text { Progeny } \\
\text { mean titre } \\
(\mathrm{u} / \mathrm{ml}) \\
F_{1}\end{array}$} & \multirow[b]{2}{*}[i]{$^{*}$} & \multirow[b]{2}{*}{$\widehat{\sigma_{G}^{2}}$} & \multirow{2}{*}{$\widehat{\sigma_{E}^{2}}$} & \multirow{2}{*}{$\widehat{\sigma_{c}^{2}} \dagger$} & \multirow[b]{2}{*}{$h^{2}$} \\
\hline & $P_{1}$ & $P_{2}$ & & & & & & \\
\hline M50 & $17 \cdot I$ & $18 \cdot 0$ & $16 \cdot 1$ & $I \cdot 49 \pm I \cdot 88$ & I $2 \cdot 26 \ddagger$ & I 5.69 & $6 \cdot 29 \ddagger$ & 0.44 \\
\hline м6o & 19.5 & I $8 \cdot 5$ & $17 \cdot 9$ & $I \cdot I 3 \pm I \cdot 58$ & $12 \cdot 02 \ddagger$ & 26.51 & 0.00 & 0.31 \\
\hline M70 & $18 \cdot 0$ & $16 \cdot 3$ & $19 \cdot 3$ & $-2 \cdot 17 \pm 2.5 \mathrm{I}$ & $16.51 t$ & $25 \cdot 77$ & 0.00 & 0.39 \\
\hline
\end{tabular}

to that in the first cycle of selection and shows a clear correlation with changes in the amount of genetic variation, $\sigma_{G}{ }^{2}$, as selection proceeds (Fig. 2). The results were again analysed using the standard form of analysis of variance (Merrick \& Caten, 1975a), and are summarized in Table 3. In both crosses where a yellow spore-colour marker was used, the yellow-spored progeny had a significantly lower mean titre than the green. When a white mutant was used (cross M52) there was no difference in titre between the spore-colour classes. Tests for epistasis in crosses M5I and M52 were again negative and hence consistent with a model of additive gene action.

The genetic variance showed a slight but not significant increase from M50 to M52. On the other hand, the variance between replicate flasks, ${\sigma_{E}}^{2}$, increased significantly over these three generations. This apparent decrease in the stability of the strains with selection is thought to be attributable to a scaling effect introduced by the logarithmic transformation used in conversion of the inhibition zone diameters to titres $(\mathrm{u} / \mathrm{ml})$.

\section{Contemporaneous comparison of strains}

The correlation between results from the contemporaneous comparison and those obtained in individual experiments during the selection programme was highly significant $(r=0.83, P<0.001)$. The two sets of data are plotted together for lines 2,4 and $2 \times 4$ in Fig. 3.

\section{DISCUSSION}

Crosses between selected lines. Segregation of alleles affecting penicillin production occurred in all three crosses between selection lines, and the genetic components of the progeny variances were as large as or larger than those for crosses between unselected wildtype isolates (Merrick \& Caten, 1975a). These observations suggest that the increases in titre had been predominantly achieved by different gene and/or allele combinations in each line. This is consistent with the use, as initial parents, of isolates belonging to different heterokaryon compatibility (h-c) groups (Merrick, 1975), since these groups are genetically diverse (Jinks et al. I966; Merrick \& Caten, I975a). Each of the initial isolates presumably carried different genes and/or alleles affecting penicillin titre, leading to the establishment of different high-titre gene combinations in each line. These results provide further evidence that variation for penicillin production in this population of $A$. nidulans isolates is determined by a large number of genes. The additive gene action found in all three crosses suggests that whilst different gene combinations had been selected in each line, these were not dependent on non-allelic interactions for their effect. These gene combinations may therefore 
$\approx \underset{0}{\forall} \stackrel{m}{0} \stackrel{m}{0}$

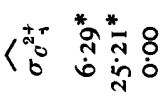

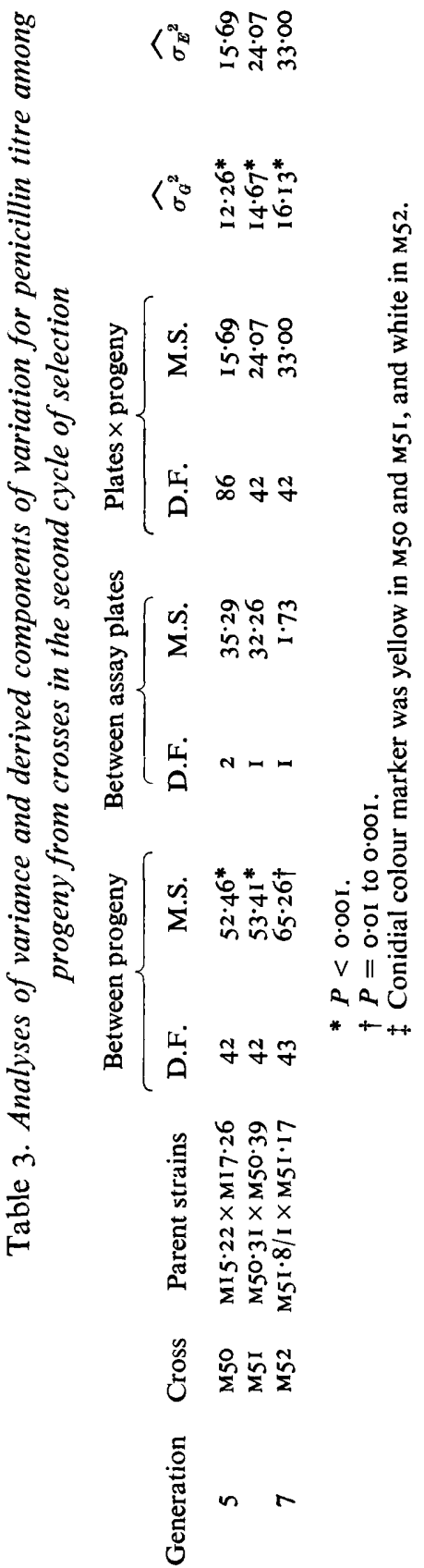



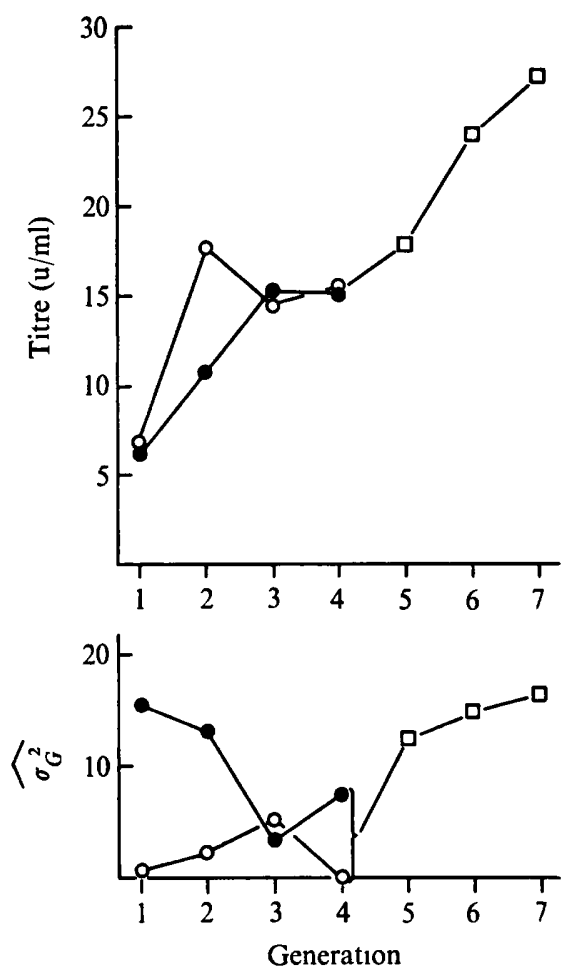

Fig. 2

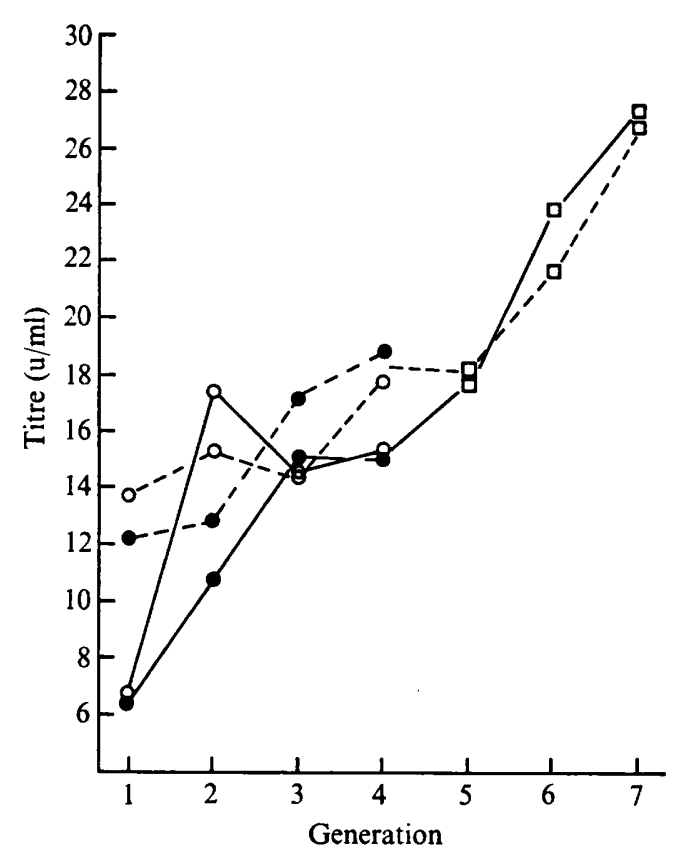

Fig. 3

Fig. 2. Response to selection for increased penicillin titre in lines 2 and 4 and renewed selection from a cross between these lines. $O$, Line $2 ; 0$, line $4 ; \square$, line $2 \times 4$.

Fig. 3. Response to selection for increased penicillin titre in lines 2, 4 and $2 \times 4$ showing the contemporaneous comparison and the accumulated response. $O$, Line $2 ; 0$, line $4 ; \square$, line $2 \times 4$. Solid line, accumulated response; broken line, contemporaneous comparison.

be broken up and the individual genes recombined additively with those selected in other lines to produce further increases in titre.

Similar crosses between lines selected for linear growth rate have been described by Simchen (1966) for monokaryons of Schizophyllum commune and by Papa (1970) for Neurospora crassa. Whilst the selection procedures in each of these studies were similar to those used for $A$. nidulans (Merrick, 1975), the results obtained on crossing between lines were different in both cases and demonstrate some of the possible outcomes of selection programmes of this type.

Tests for epistasis were not presented by Simchen (1966) or Papa (1970) but the type of gene action may be deduced from the data available in each case. Simchen's (1966) data for $S$. commune show no indication of epistasis either in the cross between high growth-rate selection lines or in any other crosses between selected material. However, Papa's (I970) results for $N$. crassa show pronounced epistasis in all three crosses between selected lines and no strains with growth rates greater than that of the faster parent were apparently recovered. This was attributed to the breakdown of polygenic complexes which had been built up in the previous selection cycle.

Information on the genetic architecture of selected strains is important when considering 
commercial strain improvement by hybridization. If non-additive gene action is present the possibility of recovering an improved recombinant strain from a 'between-line' cross may be substantially reduced, as evidenced by the results of Papa (1970) for high linear growth rate in $N$. crassa. In a situation where the gene action is mainly additive, however, improved recombinants should be more frequent. This was so in the present work, where recombinants were obtained which had a $30 \%$ increase in the titre (Fig. I) compared with the higher parent strain.

Renewed selection. The genetic variation generated by crossing between selected lines allowed a further substantial increase in titre on renewed selection. This confirms that the fall-off in response in the fourth and fifth generations of lines 2 and 4 was due to a lack of genetic variation and not to any physiological limit. The response in the second cycle of selection was similar to that in the first, but selection was not continued far enough for a second plateau to be reached (Fig. 2). It is likely that this procedure of inter-line hybridization would be repeatable for a number of cycles, with an associated stepwise increase in titre for each cycle. These results differ from those of Papa (1970), in which further selection from crosses between lines failed to exceed previous limits. This may have been due to the strains having reached a physiological upper limit so that additivity of gene action was not expressed.

Effects of genetic markers. At two points during the selection programme a white conidialcolour marker was introduced in place of a yellow one when the latter was found to show a pleiotropic depression of titre. In both instances (Tables 2 and 3 ) this change removed the difference in mean titre between spore-colours, thus indicating that the effect was associated with the $y$ gene and is not a general effect of spore-colour mutation. It is not known whether this pleiotropic effect is a property of all $y$ mutations or due to the particular mutants and/or genetic backgrounds in these experiments.

Contemporaneous comparison of strains. Such comparisons have rarely been carried out in selection programmes and are only possible when the parental material can be cloned asexually and retained until the end of the programme. The results (Fig. 3) demonstrate that the increases in titre observed in the course of the selection programme are attributable to selection of genetically improved strains and not to changes in the fermentation or assay conditions. The correlation between the contemporaneous titres and those obtained in individual experiments was increased from $r=0.76$ to $r=0.83$ when the titres were corrected against the control strains. This is in agreement with previous data (Merrick, 1975) which indicated that 'between-experiment' variation accounts for only a small proportion of the total error variation (between and within experiments). Nevertheless, the increase in the correlation after correction suggests that the use of control strains is probably advantageous.

I am grateful to Dr C. E. Caten for his advice and encouragement and for help in the preparation of the manuscript. I thank Glaxo Research Laboratories Ltd, Sefton Park, for the provision of equipment and materials, and the Glaxo staff for their continued support. A Science Research Council C.A.P.S. studentship is gratefully acknowledged. This work was further supported by grant B/RG/2196 from the Science Research Council. 


\section{REFERENCES}

Jinks, J. L., CATEN, C. E., SimChen, G. \& CRoft, J. H. (1966). Heterokaryon incompatibility and variation in wild populations of Aspergillus nidulans. Heredity 21, 227-239.

MerricK, M. J. (1975). Hybridization and selection for increased penicillin titre in wild-type isolates of Aspergillus nidulans. Journal of General Microbiology 91, 278-286.

MerRick, M. J. \& CATEN, C. E. (1975a). Inheritance of penicillin titre in wild-type isolates of Aspergillus nidulans. Journal of General Microbiology 86, 283-293.

MERRICK, M. J. \& CATEN, C. E. (1975b). The design of fermentation and biological assay procedures for assessment of penicillin production in populations of Aspergillus nidulans. Journal of Applied Bacteriology $38,121-131$.

PAPA, K. E. (1970). Inheritance of growth rate in Neurospora crassa: crosses between previously selected lines. Canadian Journal of Genetics and Cytology 12, $1-9$.

SiMCHEN, G. (I966). Monokaryotic variation and haploid selection in Schizophyllum commune. Heredity 2I, 24I-263. 